\title{
Superbeam Studies at CERN
}

\author{
A. Blondel ${ }^{\mathrm{a}}$, J. Burguet-Castell $^{\mathrm{b}}$, D. Casper ${ }^{\mathrm{c}}$, M. Donega ${ }^{\mathrm{a}}$, \\ S. Gilardoni ${ }^{\mathrm{a}}$, J. J. Gómez Cadenas ${ }^{\mathrm{b}, \mathrm{d}}$, P. Hernández ${ }^{\mathrm{d}}$ \\ M. Mezzetto ${ }^{\mathrm{e}, *}$, \\ ${ }^{a}$ Département de Physique, Université de Geneve, Switzerland \\ ${ }^{\mathrm{b}}$ IFIC, Edificio de Institutos de Paterna, Paterna, Valencia, Spain \\ ${ }^{\mathrm{c}}$ University of California at Irvine, USA \\ d CERN, Ch-1211 Geneve 23, Switzerland \\ e INFN, Sezione di Padova, Italy
}

\begin{abstract}
A conventional low energy neutrino beam of great intensity could be produced by the Super Proton Linac at CERN as a first stage of a Neutrino Factory. Water Čerenkov and liquid scintillator detectors are studied as possible candidates for a neutrino oscillation experiment which could improve our current knowledge of the atmospheric parameters $\Delta m_{a t m}^{2}, \theta_{23}$ and measure or severely constrain $\theta_{13}$. It is also shown that a very large water detector could eventually observe leptonic $\mathrm{CP}$ violation.
\end{abstract}

\section{The SPL neutrino beam}

The planned Super Proton Linac (SPL) is a $2.2 \mathrm{GeV}$ proton beam of $4 \mathrm{MW}$ power [1] which could be used as a first stage of the Neutrino Factory complex at CERN[2]. It would work with a repetition rate of $75 \mathrm{~Hz}$ delivering $1.5 \cdot 10^{14}$ protons per pulse $\left(10^{23}\right.$ protons on target (pot) in a $10^{7} \mathrm{~s}$ conventional year). Pions are produced by the interactions of the $2.2 \mathrm{GeV}$ proton beam with a liquid mercury target and focused with a magnetic horn [3]. Target and horn assumed in the following are precisely those studied for the Neutrino Factory and no optimization has been attempted for this Superbeam.

Pions next transverse a cylindrical decay tunnel of 1 meter radius and 20

\footnotetext{
* Corresponding author

Email address: Mauro.Mezzetto@pd.infn.it ( M. Mezzetto).
}

Preprint submitted to Elsevier Science 8 October 2001 
Table 1

The SPL neutrino fluxes, for $\pi^{+}$(left) and $\pi^{-}$(right) focused in the horn, computed at $50 \mathrm{~km}$ from the target.

\begin{tabular}{|c|ccc||c|ccc|}
\hline \multicolumn{5}{|c||}{$\pi^{+}$focused beam } & \multicolumn{4}{c|}{$\pi^{-}$focused beam } \\
\hline Flavor & $\begin{array}{c}\text { Absolute Flux } \\
\left(\nu / 10^{23} \mathrm{pot} / \mathrm{m}^{2}\right)\end{array}$ & $\begin{array}{c}\text { Relative } \\
(\%)\end{array}$ & $\begin{array}{c}\left\langle E_{\nu}\right\rangle \\
(\mathrm{GeV})\end{array}$ & Flavor & $\begin{array}{c}\text { Absolute Flux } \\
\left(\nu / 10^{23} \mathrm{pot} / \mathrm{m}^{2}\right)\end{array}$ & $\begin{array}{c}\text { Relative } \\
(\%)\end{array}$ & $\begin{array}{c}\left\langle E_{\nu}\right\rangle \\
(\mathrm{GeV})\end{array}$ \\
\hline$\nu_{\mu}$ & $1.7 \cdot 10^{12}$ & 100 & 0.26 & $\bar{\nu}_{\mu}$ & $1.1 \cdot 10^{12}$ & 100 & 0.23 \\
$\bar{\nu}_{\mu}$ & $4.1 \cdot 10^{10}$ & 2.4 & 0.24 & $\nu_{\mu}$ & $6.3 \cdot 10^{10}$ & 5.7 & 0.25 \\
$\nu_{e}$ & $6.1 \cdot 10^{9}$ & 0.36 & 0.24 & $\bar{\nu}_{e}$ & $4.3 \cdot 10^{9}$ & 3.9 & 0.25 \\
$\bar{\nu}_{e}$ & $1.0 \cdot 10^{8}$ & 0.006 & 0.29 & $\nu_{e}$ & $1.6 \cdot 10^{8}$ & 0.15 & 0.29 \\
\hline
\end{tabular}

meters length. These dimensions have been optimized in order to keep the $\nu_{e}$ contribution low and $\nu_{\mu}$ flux as high as possible.

We have used the MARS program[4] to generate and track pions. We have added polarization effects on $\mu$ decays and used analytical calculations to follow $\pi$ and $\mu$ decays and particle trajectories [5].

The resulting neutrino spectra are shown in Fig. 1 and Table reftab:fluxes.
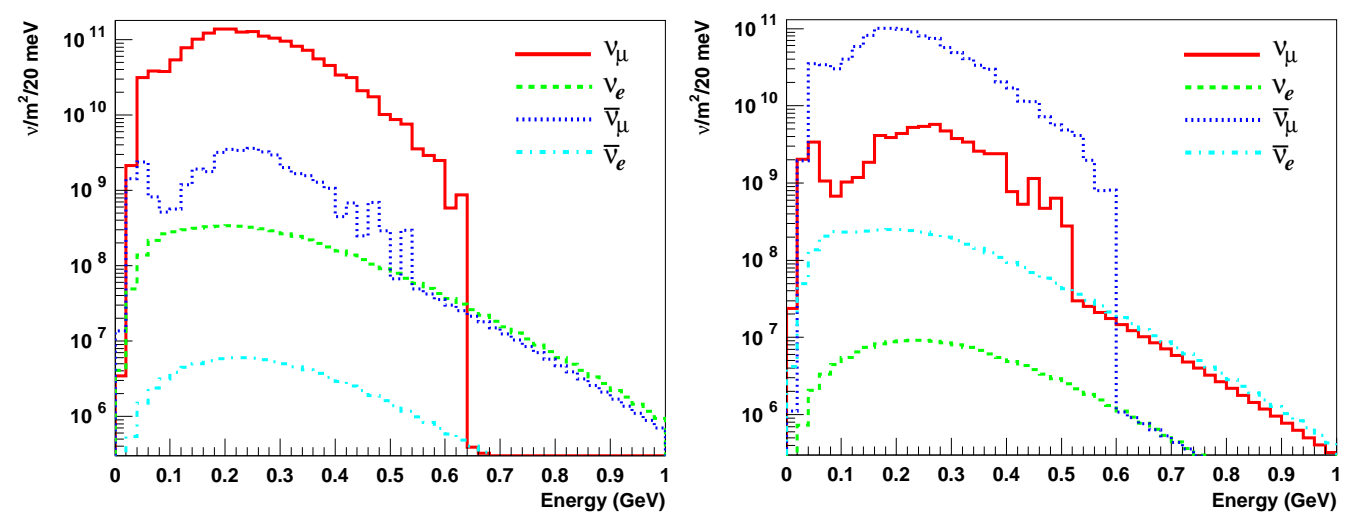

Fig. 1. The SPL neutrino spectra, for $\pi^{+}$(left) and $\pi^{-}$(right) focused in the horn, computed at $50 \mathrm{~km}$ from the target.

The average energy of the neutrinos is around $250 \mathrm{MeV}$ with a $\nu_{e}$ contamination of $\sim 0.4 \%$.

$\nu_{e}$ in the beam can be produced only by $\mu$ decays, since center-of-mass energy is below kaon production. $\nu_{\mu}$ and $\nu_{e}$ come from the same decay chain $\pi^{+} \rightarrow \nu_{\mu} \mu^{+} \rightarrow e^{+} \nu_{e} \bar{\nu}_{\mu}$ and so $\nu_{e}$ can be predicted by a direct measure of the $\nu_{\mu}$ themselves. We estimate that combining the measurement of $\nu_{\mu}$ interaction rate at the far detector with the $\nu_{\mu}$ and $\nu_{e}$ rates in a close detector $(0.5 \mathrm{kton}$ at $1 \mathrm{~km}$ from the target), the $\nu_{e}$ contamination can be established with $2 \%$ systematic and statistical errors.

\section{Detector scenarios}

Fig. 2 shows the oscillation probability $P\left(\nu_{\mu} \rightarrow \nu_{e}\right)$ as a function of the distance. Notice that the first maximum of the oscillation at $L \sim 130 \mathrm{~km}$ matches 


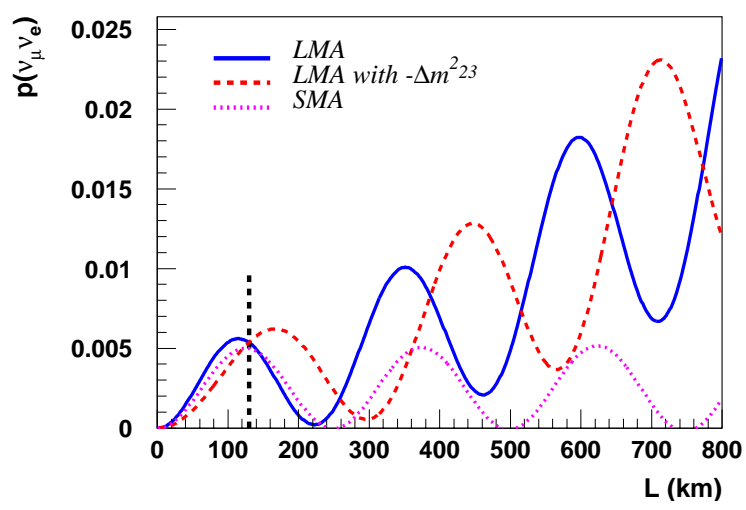

Fig. 2. $P\left(\nu_{\mu} \rightarrow \nu_{e}\right)$ computed for $\left\langle E_{\nu_{\mu}}\right\rangle=0.26 \mathrm{GeV}, \Delta m_{\text {atm }}^{2}=2.5 \cdot 10^{-3} \mathrm{eV}^{2}$, $\Delta m_{\text {sun }}^{2}=5 \cdot 10^{-5}, 6 \cdot 10^{-6} e V^{2}, \pm \Delta m_{23}^{2}, \sin ^{2} \theta_{13}=0.01, \delta=0$.

the distance between CERN and the Modane laboratory in the Frejus tunnel, where one could conceivably locate a large neutrino detector[6].

Detection of low-energy neutrinos at $O(100 \mathrm{~km})$ from the source requires a massive target with high efficiency. Moreover, a search for $\nu_{e}$ appearance demands excellent rejection of physics backgrounds, namely $\mu$ misidentification and neutral current $\pi^{0}$ production, which should be controlled to a lower level than the irreducible beam-induced background.

Backgrounds from atmospheric neutrinos remain negligible if the accumulator foreseen in the Neutrino Factory complex is used; it provides a duty cycle of 4000.

In this paper we consider two detector technologies, which have demonstrated excellent performance in the low energy regime, while being able to provide massive targets. These are, water Cerenkov detectors, as used in SuperKamiokande[7], and diluted liquid scintillator detectors as used by the LSND experiment [8] and planned for the forthcoming MiniBoone experiment[9], where both Čerenkov and scintillation light are measured.

\subsection{Water Čerenkov detectors}

We have considered an apparatus of 40 kton fiducial mass and sensitivity identical to the Super-Kamiokande experiment. The response of the detector to the neutrino beam discussed in section 1 was studied using the NUANCE[10] neutrino physics generator and detector simulation and reconstruction algorithms developed for the Super-Kamiokande atmospheric neutrino analysis. These algorithms, and their agreement with real neutrino data, have been described elsewhere[7,11,12].

The SuperKamiokande standard algorithms for $\mu / e$ separation are very effec- 
tive in the SPL-SuperBeam energy regime. ${ }^{a}$

Events with a $\pi^{0}$ are easily rejected in events where the two rings are found by a standard $\pi^{0}$ search algorithm, á la Super-Kamiokande. To reject events where only one $\gamma$ has been initially identified, an algorithm[14] forces the identification of a candidate for a second ring, which, if the primary ring is truly a single electron, is typically either very low energy, or extremely forward. By requiring that the invariant mass formed by the primary and the secondary rings is less than $45 \mathrm{MeV} / c^{2}$, almost all remaining $\pi^{0}$ contamination is removed. Being the SPL-SuperBeam more clean and at lower energies than the beam of Ref. [15], we have not attempted to introduce more aggressive $\pi^{\circ}$ rejection algorithms.

Data reduction is summarized in Table 2. Contamination by the intrinsic beam $\nu_{e}$ is dominant.

Table 2

Summary of simulated data samples a $\pi^{+}$and $\pi^{-}$focused neutrino beams. The numbers in the rightmost column (after all cuts) represent the sample used to estimate the oscillation sensitivity.

\begin{tabular}{|c|c|c|c|c|c|c|}
\hline Channel & $\begin{array}{c}\text { Initial } \\
\text { sample }\end{array}$ & $\begin{array}{c}\text { Visible } \\
\text { events }\end{array}$ & $\begin{array}{c}\text { Fit in fid. vol. } \\
\text { Single-ring } \\
100-450 \mathrm{MeV} / \mathrm{c}^{2}\end{array}$ & $\begin{array}{c}\text { Tight } \\
\text { particle } \\
\text { ID }\end{array}$ & $\begin{array}{c}\text { No } \\
\mu \rightarrow e\end{array}$ & $\begin{array}{c}m_{\gamma \gamma} \\
\leq 45 \mathrm{MeV} / \mathrm{c}^{2}\end{array}$ \\
\hline \hline \multicolumn{6}{|c|}{$\pi^{+}$focused beam } \\
\hline$\nu_{\mu}$ & 3250 & 887 & 578.4 & 5.5 & 2.5 & 1.5 \\
$\nu_{e}$ & 18 & 12. & 8.2 & 8.0 & 8.0 & 7.8 \\
$\mathrm{NC}$ & 2887 & 36.9 & 8.7 & 7.7 & 7.7 & 1.7 \\
\hline$\nu_{\mu} \rightarrow \nu_{e}$ & $100 \%$ & $82.4 \%$ & $77.2 \%$ & $76.5 \%$ & $70.7 \%$ & $70.5 \%$ \\
\hline \hline \multicolumn{7}{|c|}{$\pi^{-}$focused beam } \\
\hline $\bar{\nu}_{\mu}$ & 539 & 186 & 123 & 2.3 & 0.7 & 0.7 \\
$\bar{\nu}_{e}$ & 4 & 3.3 & 3 & 2.7 & 2.7 & 2.7 \\
$\mathrm{NC}$ & 687 & 11.7 & 3.3 & 3 & 3 & 0.3 \\
\hline $\bar{\nu}_{\mu} \rightarrow \bar{\nu}_{e}$ & $100 \%$ & $79.3 \%$ & $74.1 \%$ & $74.0 \%$ & $67.1 \%$ & $67.0 \%$ \\
\hline
\end{tabular}

\subsection{Liquid scintillator detectors}

The energy range (50 MeV-1 GeV) and the rejections against background needed by MiniBoone nicely match the requirements of our study. Neutrino${ }^{12} \mathrm{C}$ cross sections are taken from reference[16]. ${ }^{\mathrm{b}}$

Table 3 shows data reduction assuming no $\nu_{\mu}-\nu_{e}$ oscillation, for a 200 kton-

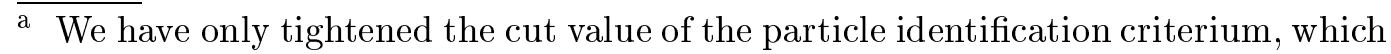
is based on a maximum likelihood fit of both $\mu$-like and e-like hypotheses $\left(P_{\mu}\right.$ and $P_{e}$ respectively). We use $P_{e}>P_{\mu}+1$ instead of the default $P_{e}>P_{\mu}$.

b They come from an upgraded version of the continuous random phase approximation method and in average they are lower by about $\sim 15 \%$ from what quoted by the MiniBoone experiment. 
Table 3

Summary of data samples in a 40 kton liquid scintillator detector at $L=130 \mathrm{~km}$ for a $200 \mathrm{kton} /$ year exposure.

\begin{tabular}{|c|c|c||c|c|c|}
\hline \multicolumn{3}{|c||}{$\pi^{+}$focused beam } & \multicolumn{3}{c|}{$\pi^{-}$focused beam } \\
\hline Channel & Initial sample & Final sample & Channel & Initial sample & Final sample \\
\hline$\nu_{\mu}^{C C}$ & 2538 & 2.5 & $\bar{\nu}_{\mu}^{C C}$ & 451 & 0.5 \\
$\nu_{e}^{C C}$ & 12 & 6 & $\bar{\nu}_{e}{ }_{C C}$ & 2.3 & 1.0 \\
$\mathrm{NC}$ (visible) & 48 & 0.5 & $\mathrm{NC}$ & 10 & $<0.1$ \\
\hline$\nu_{\mu} \rightarrow \nu_{e}$ & $100 \%$ & $50 \%$ & $\bar{\nu}_{\mu} \rightarrow \bar{\nu}_{e}$ & $100 \%$ & $50 \%$ \\
\hline
\end{tabular}

year exposure. As before, intrinsic beam $\nu_{e}$ contamination results to be the dominant background.

As one can see comparing Tables 2 and 3 the performances of both devices are quite similar. The conclusion is that one would prefer for this experiment a water detector, where truly gargantuan sizes can be afforded.

\section{Physics Potential}

\subsection{Sensitivity to the atmospheric parameters}

To illustrate the precision in measuring $\delta m_{\text {atm }}^{2}$ and $\theta_{23}$ Fig. 3 shows the result of a 200 kton-years exposure $\nu_{\mu}$ disappearance experiment on a liquid scintillator detector. The computation is performed defining 4 energy bins in the $0.1-0.7 \mathrm{GeV}$ energy range and including Fermi motion, that is by far the most limiting factor to energy reconstruction at these energies. See[17] for more details. We find that $\Delta m_{23}^{2}$ can be measured with a standard deviation of $1 \cdot 10^{-4} \mathrm{eV}^{2} / \mathrm{c}^{4}$ while $\sin ^{2} 2 \theta_{23}$ is measured at the $\sim 1 \%$ precision level.

In case of water Cerenkov, the first energy bin would be lost, being the $\mu$ below threshold. In this case the resolution on the atmospheric parameters is slightly worsened, especially in case of low $\Delta m^{2}$.

\subsection{Sensitivity to $\theta_{13}$}

The different solar solutions don't affect very much $P\left(\nu_{\mu} \rightarrow \nu_{e}\right)$ for $L=$ $130 \mathrm{~km}$ as can be seen in Fig.2. We will use SMA parameters, a hierarchical mass model, $\delta=0$. Given the low statistic and the poor energy resolution we consider in this case a counting experiment, not exploiting spectral distortions. Fig. 3(right) shows the expected sensitivity for a 5-year run with a 40 kton (400 kton) water target at a distance of $130 \mathrm{~km}$.

The sensitivity of this search results to be about 15 (60) times higher than the present experimental limit of $\sin ^{2} \theta_{13}$ coming from the Chooz experiment[18]. 

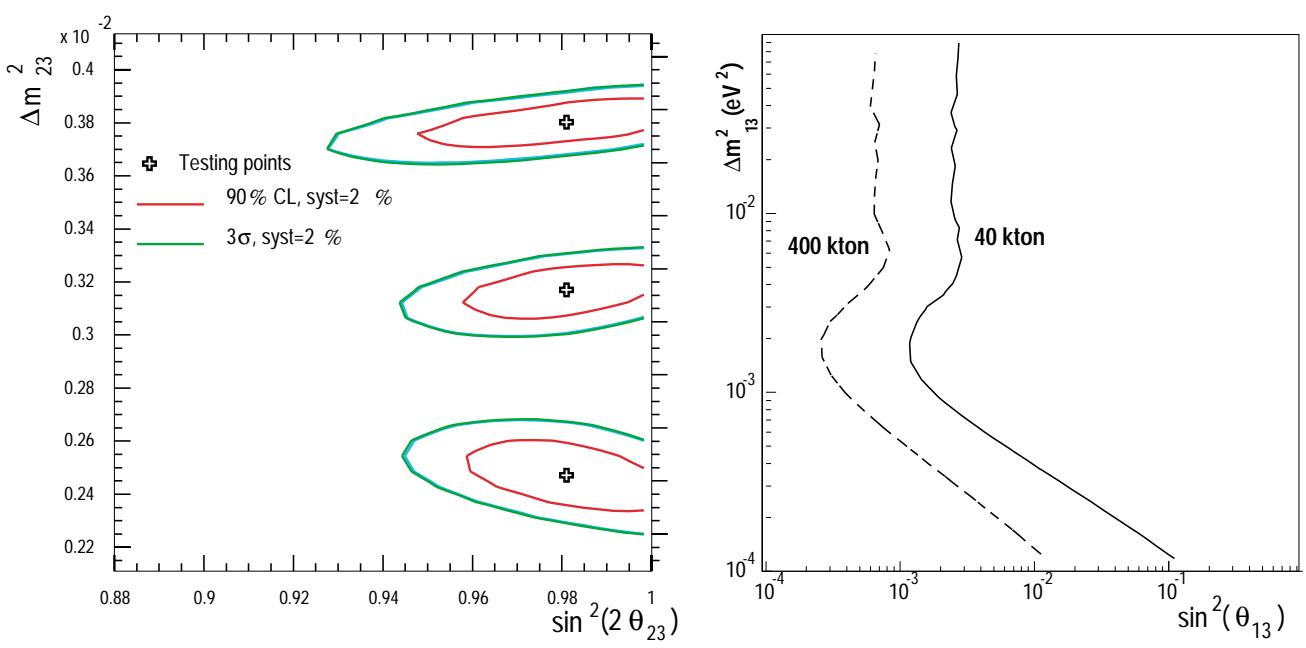

Fig. 3. LEFT: Fits of $\delta m_{\text {atm }}^{2}\left(e V^{2}\right), \sin ^{2}\left(2 \theta_{23}\right)$ after a 200 kton/year exposure, $L=130 \mathrm{~km}$, 2\% systematic errors. The crosses sign the initial points $\left(0.98,3.8 \cdot 10^{-3}\right),\left(0.98,3.2 \cdot 10^{-3}\right),\left(0.98,2.5 \cdot 10^{-3}\right)$ in $\delta m_{\text {atm }}^{2}, \sin ^{2}\left(2 \theta_{23}\right)$ coordinates. RIGHT: $\nu_{\mu} \rightarrow \nu_{e}$ oscillation sensitivity for $\pi^{+}$focused neutrino beams with a 40 (400) kton detector.

\subsection{Sensitivity to $C P$ in the $L M A-M S W$ scenario}

In the remaining of this section we will assume $\delta m_{12}^{2}=10^{-4} \mathrm{eV}^{2}$ (in the upper part of LMA) and $\sin ^{2} 2 \theta_{12}=1$.

The $\bar{\nu}_{\mu}+{ }^{16} O$ cross-section is approximately six times less than that for $\nu+{ }^{16} O$ at $E_{\nu} \simeq 0.25 \mathrm{GeV}$; to compensate that we have considered a 10 year run with focused $\pi^{-}$and a 2 year run with focused $\pi^{+}$.

We follow the approach in[19,20] and fit simultaneously the number of detected electron-like events to the $\mathrm{CP}$ phase $\delta$ and $\theta_{13}$. Notice that, although we apply a full three family treatment to our calculations, including matter effects, these are not important at the short distances and low energies considered. Fig. 4(left) shows the confidence level contours including statistical and background subtraction errors. A maximally violating $\mathrm{CP}$ phase $\left(\delta= \pm 90^{\circ}\right)$ would be just distinguishable from a non $\mathrm{CP}$ violating phase $\left(\delta=0^{\circ}\right)$.

Fig. 4(right) shows the result of the same fit, now assuming a very large water detector, such as the proposed UNO[21], with a fiducial mass of 400 kton. Clearly, the prospects to observe $\mathrm{CP}$ violation are much improved.

\section{Conclusions}

We have examined the physics potential of a low energy Superbeam which could be produced by the CERN Super Proton Linac. Water Čerenkov and liquid oil scintillator detectors have been considered. 

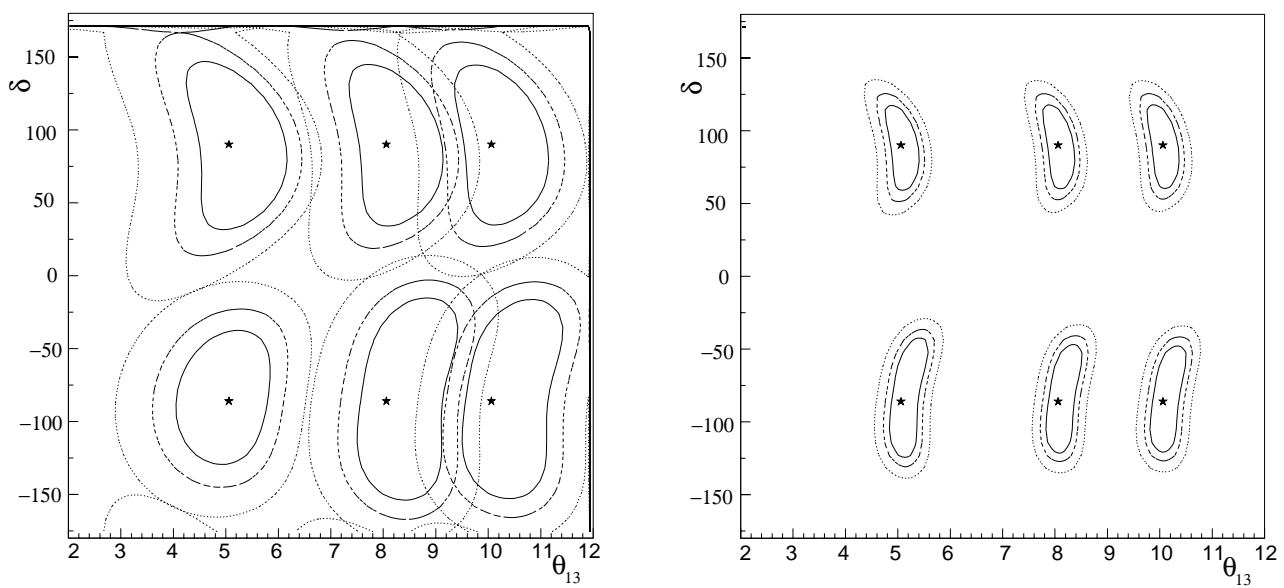

Fig. 4. $1 \sigma, 90 \% C L$ and $99 \% C L$ intervals resulting from a simultaneous fit to the $\theta_{13}$ and $\delta$ parameters. The generated values were $\theta_{13}=5^{\circ}, 8^{\circ}, 10^{\circ}, \delta= \pm 90^{\circ}$. Computed for a 10 year anti-neutrino and a 2 year neutrino run, $L=130 \mathrm{~km}$ with a 40 kton detector (left) or with a 400 kton detector (right).

The low energy of the beam studied has several advantages. Beam systematics are kept low because $2.2 \mathrm{GeV}$ protons are below the kaon production threshold. Furthermore, $e / \mu$ and $e / \pi^{0}$ separation in a water (liquid oil) detector is near optimal at these low energies. The drawback is the small anti neutrino cross section, which is more than a factor five smaller than neutrino cross section. These cross-sections are in addition quite uncertain and would certainly need to be measured.

The peak of the oscillation is at a distance of about $130 \mathrm{~km}$ where an ideal location exists, the Modane laboratory in the Frejus tunnel.

A "moderate" size detector ("only" twice as big a Super-Kamiokande) at this baseline could, in a five year run, improve the precision in the determination of the atmospheric parameters by about one order of magnitude (with respect to the expected precision of next generation neutrino experiments, such as Minos). It could also improve by more than one order of magnitude the sensitivity on $\sin ^{2} \theta_{13}$ compared to the present experimental limits.

Such a detector could also, if the solution to the solar neutrino problem lies in the upper part of the LMA, detect a maximally violating $\mathrm{CP}$ phase. For $\mathrm{CP}$ violation studies a very large detector, á la UNO (400 kton fiducial mass), is mandatory.

\section{Acknowledgements}

We wish to thank the Super-Kamiokande collaboration for allowing use of its simulation and analysis software in this study. 


\section{References}

[1] B. Autin et al., "Conceptual design of the SPL, a high-power superconducting $\mathrm{H}^{-}$linac at CERN", CERN-2000-012., Dec 2000. 89pp.

[2] B. Autin, A. Blondel and J. Ellis editors: "Prospective study of muon storage rings at CERN", CERN yellow report CERN 99-02. Home page http://muonstoragerings. cern.ch/.

[3] A. E. Ball et al. Nucl. Instrum. Meth. A 451 (2000) 359. S.Gilardoni et al. "Updated results for the Horn Study for the Nufact" note 2000/42, available at http://molat.home.cern.ch/molat/neutrino/nfnotes.html.

[4] N.V. Mokhov, Fermilab-FN-628(1995).

[5] A. Blondel, M. Donega and S. Gilardoni "Study of (Anti-)Neutrino Fluxes from a Horn Neutrino Beam Using 2.2 GeV Protons" Nufact note 2001/78. Available at http://mdonega.home.cern.ch/mdonega/lowe/homepage.html

[6] Luigi Mosca, talk given in the ECFA Neutrino Oscillation Working Group, May, 10th, 2001. Michel Spiro, talk given in the plenary session, muon days, ECFA working group, May, 12th, 2001. http://muonstoragerings.cern.ch/.

[7] Super-Kamiokande Collaboration, Phys. Lett. B 433, 9 (1998).

[8] LSND collaboration, Phys. Rev. Lett. 81, 1774 (1998).

[9] Boone proposal, http://www.neutrino.lanl.gov/BooNE/boone_proposal.ps

[10] D. Casper, private communication.

[11] M. D. Messier, UMI-99-23965.

[12] M. Shiozawa, Nucl. Instrum. Meth. A 433, 240 (1999).

[13] S. Kasuga et al., Phys. Lett. B 374, 238 (1996).

[14] T. Barszczak, University of California, Irvine Ph.D thesis (to be published).

[15] Y. Itow et al., "The JHF-Kamioka neutrino project", hep-ex/0106019

[16] E.Kolbe et al. Nucl.Phys A613 (1997) 382, Nucl.Phys A652(1999) 91.

[17] M. Mezzetto: "Neutrino Oscillations at the SPL SuperBeam", NuFact internal note 2000/60, December 2000, available at http://molat.home.cern.ch/molat/neutrino/nfnotes.html.

[18] Chooz collaboration, Phys. Lett. B 466, 415 (1999) [hep-ex/9907037].

[19] A. Cervera et al., Nucl. Phys. B579, 17 (2000).

[20] J. Burguet-Castell et al., Nucl. Phys. B 608 (2001) 301 [hep-ph/0103258].

[21] UNO Collaboration: "Physics Potential and Feasibility of UNO", preprint SBHEP01-3, available at http://superk.physics.sunysb.edu/s̃uperk/uno/ 